\title{
La médecine personnalisée : comment passer du concept à l'intégration dans un plan de développement clinique en vue d'une AMM?
}

\author{
1 Unité de Recherche Clinique, Hôpital Bicêtre, Paris, France \\ 2 Département de Pharmacologie Médicale, Faculté de Médecine, Université Lille 2, Lille, France \\ 3 Merck Serono, Lyon, France
}

Laurent Becquemont ${ }^{1}$, Régis Bordet ${ }^{2}$, Dominic Cellier ${ }^{3}$ et les participants à la table ronde $N^{\circ} 3$ de Giens XXVII

Texte reçu le 20 mars 2012 ; accepté le 4 juin 2012

\section{Mots clés :}

médecine personnalisée ; biomarqueurs ; phase de développement ; modèle économique ; Théranostic
Résumé - La personnalisation de la médecine est un des enjeux des prochaines années afin qu'à chaque patient corresponde une prise en charge individualisée. La médecine personnalisée a pour triple objectif : affiner le diagnostic, rationaliser la prise en charge thérapeutique et engager le patient dans une démarche préventive.

La personnalisation peut être caractérisée par différents descripteurs qu'ils soient liés au terrain, à la biologie, l'imagerie, au caractère lésionnel de l'entité à traiter, aux facteurs de comorbidité, aux coprescriptions, ou à l'environnement. Dans le cadre d'une médecine personnalisée centrée sur les marqueurs biologiques incluant la génétique ou la génomique l'intégration du plan de développement clinique en vue d'une autorisation de mise sur le marché (AMM) peut être segmenté en 3 temps avec un descripteur identifié avant le développement clinique, un descripteur découvert pendant le développement clinique ou un descripteur connu après le développement clinique. Pour chacun des temps il est important de bien définir les éléments d'optimisation technique, de préciser les attentes et objectifs, de regarder les aspects méthodologiques de chacune des phases du développement clinique et enfin tenir compte des aspects règlementaires très évolutifs compte tenu du peu de thérapeutiques disposant d'une AMM répondant à la définition de la médecine personnalisée ainsi que des sauts technologiques importants selon les biomarqueurs retenus et sélectionnés.

Ces considérations sont à intégrer compte tenu du temps de développement clinique en vue d'une AMM supérieur à 10 ans entre la phase précoce et l'AMM.

Par ailleurs les modèles économiques liés au contexte économique sont à prendre en compte dans les choix pour retenir ou non un biomarqueur permettant de sélectionner des populations cibles dans une population générale.

Abréviations : voir en fin d'article.

\section{Introduction}

Le but de la table ronde $\mathrm{N}^{\circ} 3$ de Giens 2011 était de répondre à la question suivante : la médecine personnalisée : comment passer du concept à l'intégration dans un plan de développement clinique en vue d'une autorisation de mise sur le marché (AMM) ?
Lors des réunions préparatoires de la table ronde il a été décidé :

- de restreindre le champ de réflexion à la personnalisation déterminée par des marqueurs biologiques incluant la génétique et la génomique. Cette focalisation a permis de se concentrer sur l'intégration des biomarqueurs compagnons ${ }^{[1,2]}$ au niveau du développement d'un médicament ou d'un dispositif médical ;

\footnotetext{
* Pour la liste des participants, voir en fin d'article.
} 
les biomarqueurs ayant déjà fait l'objet d'une précédente table ronde aux rencontres de Giens ; ${ }^{[3]}$

- de segmenter le plan de développement clinique non pas en phases 0 , I, II, III classiques du plan de développement ${ }^{[4,5]}$ en vue de l'obtention d'une autorisation de mise sur le marché mais de segmenter en 3 temps pour l'intégration du biomarqueur dans la stratégie de développement :

- $1^{\mathrm{er}}$ temps : le biomarqueur est connu en pré clinique,

- $2^{\mathrm{e}}$ temps : le biomarqueur est connu en cours de développement,

- $3^{\mathrm{e}}$ temps : le biomarqueur est connu à la fin du développement ou après l'AMM,

- d'orienter les discussions en vue de proposer des recommandations portant sur 5 items,

- les éléments techniques liés au repérage et à l'optimisation pour la réalisation du test,

- les attentes et objectifs clés dans chacun des 3 temps retenus dans le développement clinique,

- les aspects méthodologiques liés aux points clés des phases cliniques dans chacun des temps du développement,

- les aspects réglementaires liés aux points clés des phases cliniques dans chacun des temps du développement,

- les aspects économiques : spécificité ou grandes étapes liées aux 3 temps retenus dans le développement clinique.

\section{Définition de la médecine personnalisée}

La personnalisation de la médecine est un des enjeux des prochaines années afin qu'à chaque patient corresponde une prise en charge individualisée. Elle est le témoin d'un changement complet de paradigme entre une attitude commune pour tous (le blockbuster, one fits for all) à une attitude adapté aux profils de patients (the right drug for the right person). La médecine personnalisée ${ }^{[6]}$ a pour triple objectif : d'affiner le diagnostic en identifiant des marqueurs de diagnostic précoce et en permettant de définir des souspopulations de patients dont l'évolution naturelle et le pronostic sont différents ; de rationaliser la prise en charge thérapeutique en passant d'une conception de masse où les traitements sont appliqués indistinctement à l'ensemble des malades à une conception individualisée où le traitement est défini patient par patient afin d'en optimiser le rapport bénéfice/risque ; d'engager le patient dans une démarche préventive, en augmentant son adhésion et son observance tout en adaptant les programmes de prévention au profil du patient. Au-delà du concept général de médecine personnalisée, la personnalisation de la médecine recouvre trois modalités médicales opérationnelles : les stratégies préventives personnalisées, les stratégies diagnostiques personnalisées et les stratégies thérapeutiques personnalisées. Le développement d'une stratégie de médecine personnalisée peut se faire en deux temps : (i) l'identification des facteurs d'individualisation qui repose sur des études fondamentales et des études de cohortes qui permettent de mettre en évidence une association entre un facteur et une variabilité diagnostique ou thérapeutique au sein d'une population; (ii) la démonstration que l'identification d'un facteur d'individualisation à l'échelon populationnel ou individuel permet de prédire une partie de cette variabilité et d'adopter une stratégie personnalisée préventive, diagnostique ou thérapeutique. Il apparaît cependant que la première étape reste celle d'une médecine stratifiée, permettant d'identifier des sous-groupes susceptibles de répondre de manière similaire par exemple à un traitement. L'étape ultime est celle d'une médecine individualisée capable pour un malade donné, en tenant compte de plusieurs paramètres, d'adapter la stratégie diagnostique, thérapeutique ou préventive.

La personnalisation peut être sous-tendue par différents descripteurs qu'ils soient liés au terrain (âge, genre, comorbidités, profil comportemental, profil socio-économique, mode de vie), biologiques (biologie classique, génomique, protéomique), lésionnels (imagerie, anatomie pathologique), thérapeutiques (associations médicamenteuses, sédentarité, habitudes alimentaires) ou environnementaux (lieu de vie, contact avec des agents polluants, ambiance stressante...).

Dans ce contexte large de présentation et discussion de la personnalisation de la médecine la définition retenue après échange avec l'ensemble des participants de la table ronde et ce compte tenu des éléments de cadrage retenus dans l'introduction à l'issue des réunions préparatoires de Giens la définition retenue de la médecine personnalisée pour cet article est :

Moyens d'optimiser l'efficacité thérapeutique et/ou la tolérance d'un ou de plusieurs médicaments chez un patient donné par la connaissance de caractéristiques liées directement ou indirectement au statut génétique de cet individu, et/ou à une ou plusieurs anomalies du génome de lignées cellulaires responsables de sa maladie.

\section{Le biomarqueur}

\section{1. Définition}

Le biomarqueur selon la définition proposée par le National Institute of Health $(\mathrm{NIH})$ est une caractéristique qui est « une caractéristique objectivement mesurée et évaluée comme un indicateur de processus biologiques normaux ou pathologiques, ou de réponses pharmacologiques à une intervention thérapeutique $\gg .{ }^{[1,2]}$

Dans le cas du biomarqueur compagnon, celui-ci peut avoir plusieurs objectifs :

- sélection des patients cibles pour la stratification des essais cliniques ou pour la définition des catégories de patients concernés par une AMM ;

- prédiction d'une réponse thérapeutique en identifiant les répondeurs ou non répondeurs avec possibilité d'aide au choix de la dose ou de la durée du traitement ; 


\section{Réponse binaire : + ou -}

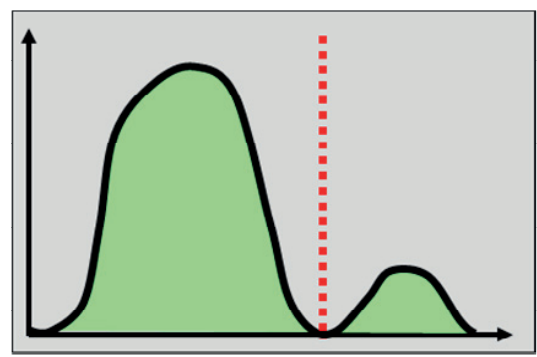

Fig. 1. Définition du seuil de positivité d'un biomarqueur.

\section{Courbe de Gauss}

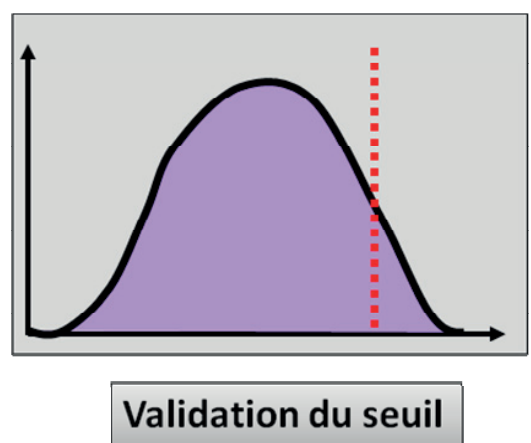

- minimisation ou prévention d'un risque médicamenteux : exemple : typage HLA chez les patients recevant un traitement exposant au risque de Syndrome de Stevens Johnson.

\subsection{Caractérisation}

Le biomarqueur retenu avant d'être intégré dans un plan de développement clinique nécessite une validation avec détermination d'un seuil fondé sur l'utilisation des courbes Receiver Operating characteristics (ROC) lorsque la valeur du biomarqueur dans une population suit une courbe de Gauss.

En matière de réponse 2 types de biomarqueurs peuvent être distingués (figure 1) : les biomarqueurs qui prédisent une réponse de type « tout ou rien » et les biomarqueurs présentant une plus ou moins grande sensibilité à un traitement donné. Le seuil peut être variable et l'identification de celui-ci peut poser des problèmes techniques.

L'évaluation du biomarqueur peut être plus complexe car dans certaines pathologies plusieurs types de biomarqueurs peuvent exister au sein de cette pathologie. Le mélanome rentre dans cette catégorie de pathologie à biomarqueur mixte de même que les cancers du sein et du poumon. La Food and Drug Administration (FDA) tient à jour une liste de biomarqueurs pharmacogénétiques d'intérêt intégrés dans les libellés des AMM. ${ }^{[7]}$

\subsection{Biomarqueurs et stratégies personnalisées}

L'évaluation de ces différentes stratégies personnalisées recouvre trois aspects :

- l'identification des descripteurs d'individualisation ;
- l'évaluation de l'effet réel de ces différents descripteurs (génétiques, biologiques, cliniques, morphologiques) d'individualisation sur ces différentes stratégies ;

- l'évaluation de l'impact de la connaissance de l'existence d'un ou plusieurs descripteurs de personnalisation sur la stratégie de prise en charge mise en œuvre par le médecin voire sur le comportement du patient.

Dans le premier cas, l'identification se fait à travers des études expérimentales, des études in silico, de cohorte, l'analyse post-hoc d'essais thérapeutiques.

Dans le second cas, il s'agit de prouver, par des études d'efficacité, qu'un paramètre qui est associé à un diagnostic ou à une variabilité de réponse à un traitement est suffisamment robuste pour changer significativement la validité du diagnostic, la prédiction d'un risque ou d'un bénéfice thérapeutique ou préventif attendu.

Dans le troisième cas, il s'agit de montrer par des études dites d'implémentation que la connaissance de ces paramètres de personnalisation modifie significativement le comportement du personnel soignant ou du patient, avec pour conséquence ou non une modification de la prise en charge et un impact sur l'organisation des soins, les paramètres médico-économiques et l'état de santé des patients.

Les deux premiers cas de figure ne posent plus de réel problème au plan méthodologique. De nombreuses études d'association ont été ou sont menées sur des populations importantes, le plus souvent à travers des consortium internationaux "genome wide Association Study" (GWAS) avec une formalisation de la démarche intégrant notamment la nécessité d'études de réplication. Des guidelines européens ont permis de formaliser les plans expérimentaux des études d'efficacité avec schématiquement deux types de plans proposés :

- un plan expérimental dans lequel les patients présentant un marqueur négatifs sont exclus et dans lequel les patients positifs sont 
Tableau I. Tableau de synthèse pour discussion et restitution table ronde : phases de développement clinique en relations avec item retenu.

\begin{tabular}{|c|c|c|c|}
\hline & $\begin{array}{c}\text { Descripteur connu avant } \\
\text { le développement } \\
\text { clinique }\end{array}$ & $\begin{array}{c}\text { Descripteur connu } \\
\text { pendant } \\
\text { le développement }\end{array}$ & $\begin{array}{l}\text { Descripteur connu à la fin } \\
\text { ou après le développement } \\
\text { clinique }\end{array}$ \\
\hline \multicolumn{4}{|c|}{ Repérage, optimisation, techniques (A) } \\
\hline \multicolumn{4}{|l|}{ Attente et objectifs (B) } \\
\hline \multicolumn{4}{|l|}{ Aspects méthodologiques (C) } \\
\hline \multicolumn{4}{|l|}{ Aspects réglementaires (D) } \\
\hline Aspects économiques (E) & & & \\
\hline
\end{tabular}

randomisés entre le groupe traité par lé candidat-médicament et le groupe recevant le placebo ou un traitement de référence ;

- un plan expérimental dans lequel la population est stratifiée en fonction du résultats du biomarqueur, chaque strate étant ensuite randomisée entre le groupe traité et le groupe placebo.

La méthodologie des études d'implémentation reste à formaliser mais cette réflexion sort du cadre de cette table ronde.

\subsection{Biomarqueurs intégrés dans les trois temps de la stratégie de développement}

Les biomarqueurs peuvent être intégrés à différents moments au plan de développement. Trois cas de figures peuvent être schématiquement distingués selon le plan tableau I défini par la table ronde :

- les biomarqueurs sont connus au début du plan de développement ;

- les biomarqueurs dont l'intérêt potentiel est découvert en cours de développement ;

- les biomarqueurs qui ne sont identifiés qu'à la fin du développement voire après l'obtention de l'AMM : la molécule peut avoir obtenu une première indication sans identification du biomarqueur puis secondairement développer un biomarqueur permettant le ciblage des patients. Exemple: Kras et Cetuximab dans l'indication cancer colorectal.

Dans le premier cas, le biomarqueur a pu être déterminé au vu des résultats d'études fondamentales, de l'identification de sousgroupes particulier au sein de cohorte mais aussi d'analyse in silico permettant grâce à une modélisation de définir un marqueur d'individualisation pertinent.

Dans la deuxième situation, c'est la mise en évidence d'une variabilité de la réponse à un candidat-médicament, avec identification d'un groupe répondeur ou non répondeur mais aussi d'un groupe avec un risque médicamenteux accru, qui conduit à analyser des marqueurs sur des échantillons sanguins ou directement au niveau tumoral dans les essais d'oncologie afin d'identifier un biomarqueur associé à cette variabilité de réponse ou de risque qui pourra être intégré aux essais thérapeutiques ultérieurs. Un exemple en est donné par l'identification de la mutation l'epidermal growth factor receptor (EGFR) comme marqueur de réponse au gefetinib/erlotinib dans le cancer du poumon.

Dans la troisième situation, le biomarqueur est identifié après la mise sur le marché voire après de nombreuses années d'utilisation d'un médicament caractérisé par une variabilité interindividuelle importante.

Des exemples récents ${ }^{[7]}$ ont été apportés par la découverte de variant génétiques communs à la variabilité de réponse et de risque médicamenteux associés aux anti-vitamines $\mathrm{K}$ ou à certains antiagrégants plaquettaires (clopidogrel).

Dans les trois situations considérées, cinq aspects du plan de développement peuvent nécessiter des réponses spécifiques (tableau II) : repérage et optimisation des techniques, attente et objectif des études, aspects méthodologiques, aspects réglementaires, aspects économiques.

\section{Synthèse de la discussion de la table ronde $\mathrm{N} \cdot 3$}

Une synthèse des discussions de la table ronde selon le plan de développement clinique segmenté en 3 temps et des 5 items retenus (tableau I), est présentée dans le tableau II.

La situation idéale est finalement lorsque le descripteur est connu dès le début du développement, en phase préclinique. Dans ce cas le développement du médicament est réalisé avec celui du test compagnon qui a le temps d'être validé avant le début des études cliniques. La méthodologie des études est alors classique. En revanche, le principal risque de cette approche est d'exclure certains patients, du fait du biomarqueur, du développement et par la suite de l'accès au médicament commercialisé. Autant cela est aisé lorsque le descripteur donne une réponse tranchée (répondeur/non répondeur), autant la situation se complexifie lorsque le descripteur caractérise la population sous une forme Gaussienne unimodale (panel de droite de la figure 1) : on fixe alors un seuil subjectif qui 
Tableau II. Phases de développement clinique en relations avec item retenu : réflexions issues de la table ronde $\mathrm{N}^{\circ} 3$.

\begin{tabular}{|c|c|c|c|}
\hline & $\begin{array}{l}\text { Descripteur connu avant } \\
\text { le développement clinique }\end{array}$ & $\begin{array}{l}\text { Descripteur connu pendant } \\
\text { le développement }\end{array}$ & $\begin{array}{l}\text { Descripteur connu à la fin } \\
\text { ou après le développement clinique }\end{array}$ \\
\hline $\begin{array}{l}\text { Repérage, optimisation, } \\
\text { techniques (A) }\end{array}$ & $\begin{array}{l}\text { Cible/physiopathologie } \\
\text { Métabolisme } \\
\text { Tests in vitro } \\
\text {. Validation biologique du test }\end{array}$ & $\begin{array}{l}\text { Analyse en sous-groupe/variabilité } \\
\text { de la réponse/identification des fac- } \\
\text { teurs de variabilité } \\
\text {. Nature du marqueur d'individualisa- } \\
\text { tion à adapter selon l'aire thérapeu- } \\
\text { tique }\end{array}$ & $\begin{array}{l}\text {. Analyse en sous-groupe/variabilité de la } \\
\text { réponse/identification des facteurs de } \\
\text { variabilité } \\
\text {. Apparition d'un effet indésirable } \\
\text { majeur } \\
\text {. Nature du marqueur d'individualisation } \\
\text { à adapter selon l'aire thérapeutique }\end{array}$ \\
\hline Attente et objectifs (B) & $\begin{array}{l}\text {. Go/no Go en phase I : } \\
\text { identification de la population des } \\
\text { répondeurs } \\
\text {. Validation de l'intérêt du test }\end{array}$ & $\begin{array}{l}\text {. Validation de l'existence d'un sous- } \\
\text { groupe cible } \\
\text {. Optimisation du biomarqueur }\end{array}$ & $\begin{array}{l}\text {. Compréhension de l'effet observé : } \\
\text { absent, moderé, très important } \\
\text {. Compréhension de l'absence de } \\
\text { réponse dans un sous-groupe } \\
\text {. Exclusion des patients à risque : objectif } \\
\text { de protection } \\
\text {. Prise en compte d'une nouvelle infor- } \\
\text { mation scientifique (évolution très rapide } \\
\text { des avancées scientifiques) }\end{array}$ \\
\hline $\begin{array}{l}\text { Aspects } \\
\text { méthodologiques }(\mathrm{C})\end{array}$ & $\begin{array}{l}\text {. Test : fiabilité, reproductibilité, vers } \\
\text { accréditation du test } \\
\text {. Design de l'étude : } \\
\text { Phase I : introduction du test pour } \\
\text { valider sa mise en œuvre/extension vers } \\
\text { d'autres indications (en onco) } \\
\text { Phases II et III } \\
\text { Test binaire : sélection des patients } \\
\text { Test +/- : stratification selon réponse } \\
\text { (seuil de réponse) }\end{array}$ & $\begin{array}{l}\text {. Phase précoce : identification des } \\
\text { sous-groupes de patients/méthodes } \\
\text { adaptatives } \\
\text { Phase tardive prospective : sélection } \\
\text { et stratification des patients } \\
\text { Développement technologique du } \\
\text { biomarqueur (faisabillité, standardis- } \\
\text { ation, fiabilité) } \\
\text {. Études d'implémentation prospec- } \\
\text { tives }\end{array}$ & $\begin{array}{l}\text {. Études post-hoc } \\
\text {. Phase III : pas de nouvel essai lié à des } \\
\text { problèmes de coût de développement } \\
\text {. Études d'implémentation } \\
\text { rétrospective (exemple : AVK) } \\
\text { Études prospectives : très importantes } \\
\text { dans ce type de développement clinique }\end{array}$ \\
\hline
\end{tabular}


Tableau II. Suite.

\begin{tabular}{|c|c|c|c|}
\hline & $\begin{array}{l}\text { Descripteur connu avant } \\
\text { le développement clinique }\end{array}$ & $\begin{array}{l}\text { Descripteur connu pendant } \\
\text { le développement }\end{array}$ & $\begin{array}{l}\text { Descripteur connu à la fin } \\
\text { ou après le développement clinique }\end{array}$ \\
\hline Aspects réglementaires (D) & $\begin{array}{l}\text { Si autorisation d'AMM : évolution } \\
\text { vers évaluation des tests et marquage } \\
\mathrm{CE} \\
\text {. Avis scientifique recommandé en } \\
\text { amont de la demande AMM } \\
\text { Stratégie industrielle : couplage ou } \\
\text { non du nouveau médicament de la } \\
\text { classe et du biomarqueur selon son } \\
\text { caractère innovant } \\
\text { Accompagnement et veille régle- } \\
\text { mentaire : évolution de la technologie }\end{array}$ & $\begin{array}{l}\text { Si autorisation d' AMM évolution vers } \\
\text { évaluation des tests et marquage CE } \\
\text {. Questionnement sur la prise en } \\
\text { charge des patients avec biomarqueur } \\
\text { négatif } \\
\text {. Avis scientifique en cours de dével- } \\
\text { oppement avec validation du test à la } \\
\text { vue des données cliniques } \\
\text { Stratégie industrielle : couplage ou } \\
\text { non du nouveau médicament de la } \\
\text { classe et du biomarqueur selon son } \\
\text { caractère innovant } \\
\text { découplage }\end{array}$ & $\begin{array}{l}\text { Avis des autorités : études post-hoc ou } \\
\text { études faites à la demande des autorités } \\
\text { réglementaires (validation du plan de } \\
\text { développement) } \\
\text { Reproduction de l'analyse post-hoc sur } \\
\text { d'autres essais } \\
\text { Questionnement sur la prise en charge } \\
\text { des patients avec biomarqueur négatif }\end{array}$ \\
\hline Aspects économiques (E) & $\begin{array}{l}\text { Possible réorganisation industrielle: } \\
\text { partenariat public-privé ou intégra- } \\
\text { tion dans la stratégie pharmaceutique } \\
\text { (acquisitions de start up) } \\
\text {. Prise en compte dans la fixation du } \\
\text { prix du biomarqueur (valorisation du } \\
\text { fait de la restriction de la cible traitée : } \\
\text { patient répondeur) }\end{array}$ & $\begin{array}{l}\text { Réserver le médicament à la popula- } \\
\text { tion qui en bénéficie le plus puis fixer } \\
\text { ou adapter le prix } \\
\text {. Prix différencié en fonction des } \\
\text { sous-groupes } \\
\text { (contraintes économiques liées au } \\
\text { contexte à évaluer pays par pays) }\end{array}$ & $\begin{array}{l}\text {. Réserver le médicament à la population } \\
\text { qui en bénéficie le plus puis fixer ou } \\
\text { adapter le prix } \\
\text {. Prix différencié en fonction des sous- } \\
\text { groupes (contraintes économiques liées } \\
\text { au contexte à évaluer pays pas pays) }\end{array}$ \\
\hline
\end{tabular}

AMM : autorisation de mise sur le marché ; AVK : anti-vitamines K

va exclure une partie de la population qui certes aura une moins bonne réponse mais une réponse tout de même. Dans le cas d'un nouveau médicament sans autres options thérapeutiques, le biomarqueur peut poser des problèmes éthiques. Pour l'instant les descripteurs obtenus très précocément sont essentiellement ceux prédisant la pharmacocinétique des médicaments (via leur métabolisme génétiquement déterminé par les cytochromes P450 ou leur transport).

La situation intermédiaire dans laquelle le descripteur est identifié en cours de développement est plus délicate. Elle peut d'une part permettre de «sauver » un candidat médicament qui n'aurait pas obtenu son AMM sans segmentation de la population répondeuse (exemple du gefetinib dans le cancer du poumon) ; la plupart des thérapies ciblées (inhibiteurs de tyrosine kinase) dans le cancer sont maintenant développées de la sorte. En revanche, les aspects méthodologiques sont encore très flous. En effet, dans ce deuxième cas de figure, on ne dispose pas d'un essai randomisé sur le biomarqueur. L'étude de phase III est en route ou juste terminée et le biomarqueur est en fait testé à posteriori (étude post hoc) sur les collections biologiques. Autant cette approche serait considérée comme irrecevable dans le développement classique d'un candidat médicament, autant dans le domaine de l'oncologie, elle est presque sur le point de devenir une habitude, conduisant à l'AMM. Une réflexion sur la nécessité d'études complémentaires est indispensable. ${ }^{[8,9]}$

La dernière situation dans laquelle le descripteur est identifié après la mise sur le marché est pour l'instant la plus fréquente. Des exemples intéressants s'accumulent concernant les loci HLA de susceptibilité à des accidents immunoallergiques (Lyell, drug reaction with eosinophilia and systemic symptoms [DRESS] syndrome) qui permettent déjà pour la prescription de certains médicaments comme l'abacavir d'exclure les sujets à risque d'EIG.

Il convient cependant, comme dans la première situation, de ne pas exclure trop vite les patients pour un traitement donné : l'exemple du clopidogrel pour lequel un biomarqueur génétique de bioactivation a été identifié 10 ans après sa commercialisation est 
assez illustratif : on arrive avec un génotypage à identifier des « bons répondeurs » et des «moins bons répondeurs » pour lesquels on ne sait pas très bien l'ampleur du bénéfice clinique qu'ils retirent, mais qui doivent probablement en retirer un (même faible). En l'absence d'alternative au clopidogrel, ces « moins bons répondeurs » devraient toujours avoir accès au traitement sous peine d'une perte de chance. Fort heureusement, pour ces « moins bons répondeurs », il existe une alternative thérapeutique permettant d'utiliser le biomarqueur pharmacogénétique pour le bénéfice du patient, sans risque de perte de chance.

Dans ce troisième cas de figure, sur le plan méthodologique, on ne dispose que d'études rétrospectives d'essai ou au mieux des cohortes prospectives dépourvues de groupe contrôle ce qui implique bien évidemment des biais et nécessite de nombreuses réplications des résultats avant de pouvoir se fier à ces descripteurs découverts après l'AMM.

Enfin, quel que soit le cas de figure, l'aspect du financement du biomarqueur pose toujours problème : son prix doit-il être intégré à celui du médicament ? Est-ce au groupe pharmaceutique de le prendre en charge ? Ce grand flou habituel sur le financement des biomarqueurs est pourtant toujours réglé de la même manière : pour les biomarqueurs de réponse tumorale, c'est l'Institut national du cancer (INCA) qui a financé des plateformes pour permettre l'accès des patients à ces tests. Quand un médicament est contre indiqué en cas d'insuffisance rénale ou nécessite des contrôles réguliers de transaminases ou d'international normalized ratio (INR), c'est notre système de santé qui prend en charge, on ne voit pas pourquoi, pour les nouveaux biomarqueurs cela devrait être différent, pour peux que le patient en tire un bénéfice substantiel.

\section{Recommandations}

\subsection{Assurer la veille réglementaire}

Compte tenu de l'évolution et des sauts technoloqiques rapides au cours du développement clinique en vue de l'obtention d'une $\mathrm{AMM}$, il est recommandé d'assurer une veille réglementaire régulière afin d'adapter les modèles de plans de développement en fonction de l'évolution des réglementations. La table ronde $\mathrm{N}^{\circ} 3$ bien qu'ayant limité son périmètre d'analyse aux marqueurs biologiques incluant la génétique et la génomique recommande de suivre l'évolution des recommandations européennes (Agence européenne du médicament ou EMA) et américaines (FDA). Selon les stades du développement clinique et le niveau de connaissance du biomarqueur l'impact des réglementations peut être variable. Dans le cas où le descripteur est connu avant le développement clinique celui-ci doit être inclus comme facteur discriminant dans l'ensemble des études cliniques. Lorsque le descripteur est découvert pendant le développement clinique l'impact de l'évolution de la réglementation est à mesurer au cas par cas. Des analyses en sous-groupes de patients définis selon la présence ou non des marqueurs prédictifs devront être réalisées dans le plan de développement. La prise en compte des écarts de réglementations entre les instances réglementaires européennes, américaines et autres réglementations inhérentes aux pays concernés peuvent influer sur les stratégies de développement avec pour conséquence une mise à disposition d'AMM de façon non contemporaine.

\subsection{Disposer d'un biomarqueur en pré clinique ou en phase précoce du développement}

L'identification d'un biomarqueur de façon très précoce permet de définir le profil des patients répondant le mieux à la thérapie concernée. Le biomarqueur identifié au regard des résultats des études fondamentales permet d'optimiser le profil des patients. $\mathrm{La}$ modélisation peut être réalisée à ce stade ce qui permet de réduire le nombre de patients dans les plans de développement d'où une économie de temps dans le développement de la molécule jusqu'à obtention de l'autorisation de mise sur le marché. La table ronde $\mathrm{N}^{\circ} 3$ compte tenu à ce jour du peu d'exemples de développement complet incluant un biomarqueur dès la phase précoce jusqu'à la mise à disposition du produit a attiré l'attention sur le risque de perte de candidat valeureux à l'éligibilité thérapeutique liée à l'exclusion trop précoce de molécules identifiées et retenues sur la base d'un biomarqueur.

\subsection{Définir un modèle économique}

Au regard des expériences actuelles pour le nombre restreint de produits ayant bénéficié d'une autorisation de mise sur le marché intégrant un biomarqueur, le groupe de travail recommande la tenue d'une table ronde spécifiquement dédiée aux impacts économiques des produits intégrant un biomarqueur dans leur développement.

Plusieurs questions se posent autour du modèle économique telles que :

- le coût du test associé à la molécule : parle-t-on d'un coût global molécule plus test (le médicament étant considéré comme une entité globale permettant la prise en charge du coût du médicament plus celle de la réalisation du test) ?

- le coût du médicament et de sa valorisation économique : le ciblage des patients réduisant le nombre de patients concernés par le traitement justifie-t-il une valorisation du coût du traitement ?

- la conjoncture économique en France mais aussi celle de l'ensemble de la Communauté européenne limitant les enveloppes globales consacrées aux prises en charge thérapeutiques impose une réflexion sur les conséquences économiques. En ce qui concerne le traitement du cancer la personnalisation du traitement fait-elle changer le modèle économique des prises en charge actuelles ? 


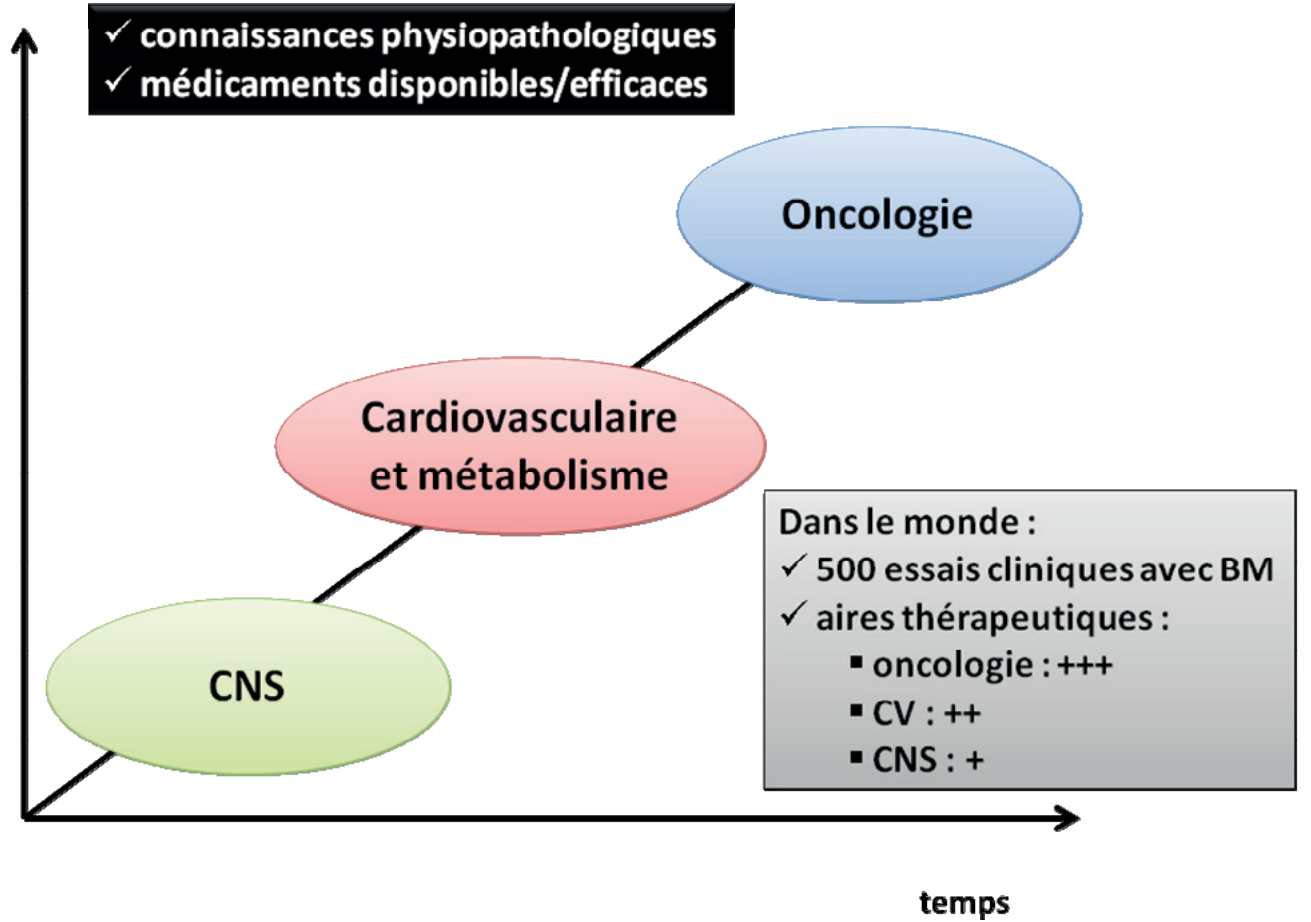

Fig. 2. Aires thérapeutiques et état des connaissances biomarqueurs médecine personnalisée.

- dans le cadre de l'exemple du traitement du cancer le passage d'un traitement aigu à une chronicité de la maladie grâce aux avancées thérapeutiques doit-il intégrer cette dimension dans les nouveaux business model?

Ces questions soulevées lors de la table ronde ont justifié la tenue ultérieure d'une table ronde spécifique modèle économique spécifique pour les traitements entrant dans le cadre de la médecine personnalisée.

Le groupe a attiré l'attention sur le fait que les questions étaient restrictives en raison de la limitation du périmètre de travail restreint aux marqueurs biologiques incluant la génétique et la génomique. Un champ important de la discussion sur le modèle économique pourrait être couvert sur les outils technologiques permettant d'optimiser la médecine personnalisée.

\subsection{Ouvrir les champs thérapeutiques à d'autres aires thérapeutiques que l'oncologie}

L'observation tirée d'une étude biomarqueurs réalisée par L'Alliance pour la recherche et l'innovation des industries de santé (ARIIS) ${ }^{[10]}$ montre aujourd'hui 500 essais cliniques associant un biomarqueur dans les plans de développement clinique en vue de l'obtention d'une autorisation de mise sur le marché. Ces essais cli- niques couvrent l'ensemble des 3 temps que le groupe a retenus dans l'intégration de la stratégie de développement. Que le biomarqueur soit connu dès la phase pré clinique (temps 1) ou au cours du développement (temps 2) ou même à la fin du développement (ou après l'AMM) [temps 3]. Les aires thérapeutiques principalement concernées sont l'oncologie, le cardio-vasculaire et le système nerveux central.

Aujourd'hui que ce soit sur l'ensemble des connaissances précoces sur la recherche de biomarqueurs ou les médicaments intégrant un biomarqueur disponibles en thérapeutique, l'oncologie reste le domaine précurseur donc le plus avancé de ce type de recherche.

\subsection{Réagir à des voyants pharmacologiques ou cliniques en cours de développement}

Le groupe de travail recommande du fait des expériences acquises sur plusieurs molécules de relever des voyants cliniques ou pharmacologiques très précocement comme par exemple : effet immuno allergique en cours de développement, détection d'apparition mutation résistance (exemple de variabilité de réponse et de risques médicamenteux associés aux anti vitamines $\mathrm{K}$ ou à certains anti agrégants plaquettaires (clopidogrel). 


\subsection{Garantir l'accès au test}

L'introduction du test pour la sélection des patients à l'éligibilité d'un traitement pose des problèmes :

- de réalisation liés à la formation technique des équipes d'où certaines inégalités possibles dans la prise en charge des patients ;

- de fiabilité dans la reproductibilité des résultats, ceux-ci étant discriminants dans l'éligibilité des patients à une thérapeutique répondant aux tests ;

- dans la rapidité liée à la mise à disposition des résultats avant l'introduction de la thérapeutique choisie selon la réponse aux tests ;

- de financement lié à la mise à disposition de plateforme et d'équipe permettant la prise en charge pour la réalisation du test.

\subsection{Mutualisation des collections biologiques}

La médecine personnalisée évoluant très rapidement, la découverte de nouveaux biomarqueurs nécessite la mise à disposition et la protection du matériel biologique. Le nombre de technologies et biomarqueurs grandissant favorise l'augmentation de recherche sur du matériel parfois ancien et limité. La mutualisation des collections biologiques est un moyen de répondre à ces demandes croissantes tout en permettant de centraliser à la fois les échantillons mais aussi l'analyse des résultats.

\section{Conclusions}

La médecine personnalisée ouvre de nouvelles perspectives et voies de recherche en vue de mettre à disposition de nouvelles molécules ou de nouvelles innovations technologiques. La finalité est d'obtenir de véritables solutions thérapeutiques personnalisées afin d'optimiser la prise en charge thérapeutique des patients. Ces nouvelles voies de recherche imposent non seulement une mise à niveau des acteurs sur le plan du vocabulaire et de la compréhension de ces nouvelles approches thérapeutiques mais aussi la création d'interfaces entre l'univers pharmaceutique et techniques industrielles.

La médecine personnalisée pose les problèmes liés au développement de molécules classiques mais aussi ceux liés à la technologie des tests en lien avec le biomarqueur.

La prise en compte de la fiabilité de la mesure du test de la standardisation de la technologie et de l'accessibilité aux techniques est importante dans la mise au point de traitements répondant au concept de médecine personnalisée.

La personnalisation de la médecine enjeu de ces prochaines années doit permettre à chaque patient de trouver la meilleure prise en charge ou solution thérapeutiques. Toutefois même s'il est important pour le futur de réfléchir à ce que chaque patient puisse bénéficier d'une alternative thérapeutique, il est important de considérer qu'un ciblage trop précoce risque d'exclure des molécules potentiellement valeureuses. Pour conclure, les impacts économiques de cette nouvelle approche, enjeu des prochaines années, doivent être pris en considération très tôt afin de valoriser afin de disposer de moyens techniques et financiers pour la mise à disposition de nouvelles thérapeutiques entrant dans le champ de la médecine personnalisée.

\section{Participants.}

Marc Bardou (CIC, Dijon), Philippe Beaune (Hôpital européen Georges Pompidou, Paris), Chantal Belorgey (Afssaps, Saint Denis), Frédéric Bibeau (CRLC Val d'Aurelle, Montpellier), Jean-Pierre Boissel (UCBL, Tassin la demi Lune), Soizic Courcier (GlaxoSmithKline), Frédérique De Broucker (Medtronic), Jacques Demotes-Mainard (MESR, Paris), Isabelle Diaz (Leem, Paris), Jean-Yves Fagon (CEPS, Paris), Danièle Girault (Novartis Pharma), Sylvia Goni (Laboratoire Lundbeck), Rémy Hanf (Genfit, Loos), Marie-Annick Hermann (Bristol Myers Squibb), Patrick Mismetti (CHU, Saint-Étienne), Monique Morali (Merck Serono), Mira Pavlovic (HAS, Saint Denis la Plaine), Elena Perrin (Lilly France), Yannick Plétan (Pfizer), Christophe Sauce (Boehringer Ingelheim), Gilles Vassal (IGR, Villejuif).

\section{Conflits d'intérêts. Aucun.}

Abréviations. AMM : autorisation de mise sur le marché; DRESS syndrome : drug reaction with eosinophilia and systemic symptoms syndrome;EIG: effets indésirables graves;EMA: Agence européenne du médicament ; FDA : Food and Drug Administration; GWAS : genome-wide Association Study; INCA : Institut national du cancer ; INR : international normalized ratio ; NIH : National Institute of Health; ROC : receiver operating characteristics.

\section{Références}

1. ANSM biomarqueurs et produits de santé. 2011. http://ansm.sante.fr/Activites/Biomarqueurs/Biomarqueurs-et-produits-de-sante/(offset)/0

2. Biomarqueur: definition working group: biomarkers and surrogate endpoints: preferred definitions and conceptual framework. Clin Pharmacol Ther 2001; 69 (3): 89-95

3. Gueyffier F, Dib M, Boissel JP. Biomarqueurs - Utilisation au cours du développement et pour l'enregistrement des médicaments Biomarqueurs : utilisation au cours du développement et pour l'enregistrement des médicaments. Therapie 2001; 56(4): 355-61

4. FDA. Drug development and aproval proces. http://www.fda.gov/Drugs/ DevelopmentApprovalProcess/default.htm

5. EMA. Clinical efficacy and safety guidelines. http://www.ema.europa.eu/ ema/index.jsp?curl=pages/regulation/general/ general_content_000085.jsp\&mid=WCOb01ac0580027549

6. EMA. Personalized medicine. http://www.ema.europa.eu/ema/ index.jsp?curl=pages/special_topics/general/ general_content_000347.jsp\&mid=WC0b01ac05800baeda

7. FDA. Pharmacogenomic biomarkers in drug labels. http://www.fda.gov/ Drugs/ScienceResearch/ResearchAreas/Pharmacogenetics/ ucm083378.htm 
8. EMA. Reflection paper on methodological issues associated with pharmacogenomic biomarkers in relation to clinical development and patient selection. http://www.ema.europa.eu/ema/index.jsp?curl=pages/regulation/ general/general_content_000411.jsp\&mid=WC0b01ac058002958e

9. EMA. Concept paper on key aspects for the use of pharmacogenomic methodologies in the pharmacovigilance evaluation of medicinal products. http:// www.ema.europa.eu/ema/index.jsp?curl=pages/regulation/general/

general_content_000411.jsp\&mid=WCOb01ac058002958e
10. ARIIS. Le biomarqueur comme outil de diagnostic compagnon de produits thérapeutiques. 2010. http://www.ariis.fr/wp-content/uploads/2011/03/ biomarqueurs-etude-version-complete-130-slides.pdf

Correspondance et offprints : Dominic Cellier, Laboratoire Merck Serono, 37 rue Saint Romain, 69379 Lyon Cedex 08, France.

E-mail : dominic.cellier@merckgroup.com 